\title{
Peripheral blood levels of cellular and humoral immunity parameters in esophageal and gastric cancer patients
}

\author{
HUSEYIN BEGENIK', MEHMED KURSAD TURKDOGAN ${ }^{2}$ AHMET CUMHUR DULGER, \\ MEHMET NACI ALDEMIR', RAMAZAN ESEN ${ }^{l}$, RAFET METE
}

${ }^{1} Y u z u n c u$ Yil University, Medical Faculty, Department of Internal Medicine, Van, Turkey

${ }^{2}$ Bezmi Alem University, Medical Faculty, Department of Gastroenterology, Istanbul, Turkey

${ }^{3}$ Yuzuncu Yil University, Medical Faculty, Department of Gastroenterology, Van, Turkey

${ }^{4}$ Namik Kemal University, Medical Faculty, Department of Gastroenterology, Tekirdag, Turkey

\begin{abstract}
Aim: To evaluate parameters of cellular and humoral immunity in gastric and esophageal cancer patients.

Material and methods: In this study, we recruited 40 patients with newly diagnosed esophageal cancer (20 of them) or stomach cancer (20 of them) and 20 healthy individuals of similar age as a control group. In the study and control groups we measured CD3, CD4, CD8, CD19, CD4/CD8 ratio and the ratio of natural killer cells using the flow cytometer device. Results were evaluated with factorial analysis of variance and Duncan test.

Results: The CD3 and CD8 ratios were significantly higher in patients with esophageal cancer ( $p=0.012, p=0.003$, respectively) and the ratio of NK cells was significantly higher in patients with stomach cancer $(p=0.001)$ when compared to the control group. The ratio of CD19 was significantly lower in the two cancer groups $(p=0.031)$. There were no significant differences in the ratio of CD4 and CD4/CD8 between the groups. No correlation between the stage of cancer and the ratio of CD cells was detected.

Discussion: In conclusion, understanding of the cancer immunology of esophageal and stomach cancer will provide insight into the pathogenesis of the cancer. Therefore, further and extensive cancer immunology studies should be conducted to understand the nature of the upper gastrointestinal tract cancers.
\end{abstract}

Key words: esophageal cancer, gastric cancer, cellular and humoral immunity.

(Centr Eur J Immunol 2013; 38 (3): 355-357)

\section{Introduction}

Carcinogenesis is related to several factors such as genetic abnormalities, diet, lifestyle, and immune deficiencies [1-6]. Cancer patients have different immune responses. Tumor-induced immunosuppression is the main problem in cancer cell biology $[7,8]$. In the case of cancer, many immunologic alterations occur in the lymphocyte subgroups including CD4, CD8, B-lymphocytes and natural killer (NK) cells. If patients with cancer have impaired lymphocyte functions, the prognosis is usually poor [9].

Patients with gastrointestinal cancer have also immune system alterations including decreased T-cells proliferation and CD4/CD8 ratio, and diminished T-helper cell cytokine production [10].

In this study, we aimed to investigate the changes of humoral and cell-mediated immune system functions involving peripheral blood lymphocyte groups and subgroups in patients with newly diagnosed esophageal and stomach cancer.

\section{Material and methods}

In this study, we recruited 40 patients with newly diagnosed esophageal cancer (20 of them) or stomach cancer

Correspondence: Dr. Huseyin Begenik, Yuzuncu Yil University, Medical Faculty, Department of Internal Medicine, Van, Turkey, tel. +90-(432)-215 0473, fax +90-(432)-216 7519, e-mail: hbegenik@gmail.com 
(20 of them), while age-matched 20 healthy individuals were recruited as a control group. The diagnosis of cancer was made by histopathologic examination of the endoscopic biopsy materials. The patients who were previously diagnosed with cancer or received any cancer-related treatment such as surgery, chemotherapy, or radiotherapy were excluded from the study.

CD3, CD4, CD8, CD4/CD8 ratio, CD 19 and NK cell's antigens $\left(\mathrm{CD}^{-}, \mathrm{CD} 16^{+}\right.$, and $\left.\mathrm{CD}^{-} 6^{+}\right)$were studied in all cases. For this purpose, $2 \mathrm{cc}$ blood samples were collected into the standard cell count tubes with ethylene diamine tetraacetic acid (EDTA). Red blood cells were eliminated from the samples with immunoprep solution using Coulter Q-Prep device. Subsequently, $100 \mu \mathrm{l}$ of the yielded solutions containing mononuclear cells were mixed with $20 \mu \mathrm{l}$ of the combination of the antihuman antiCD $3 \mathrm{IgG}_{1}$ (FITC), antiCD4 $\mathrm{IgG}_{1}$ (FITC), antiCD8 $\mathrm{IgG}_{1}$ (PE), antiCD16 $\mathrm{IgG}_{1}$ (PE), antiCD19 $\mathrm{IgG}_{1}$ (PE) and antiCD56 $\mathrm{IgG}_{1}$ (PE) monoclonal antibodies. Then, positive cells were counted with a flow cytometry device (Coulter Epics XL).

Statistical data analysis was performed with SPSS 15.0 software program. The yielded data including age, CD3, CD4, CD8, CD4/CD8, CD19 and NK cells were correlated with the cancer types and sex with factorial variance analyses (Factorial ANOVA). The factorial analysis of variance was used to analyze the interactive effects of multiple categorical independent variables. When significant differences among main effects were observed using ANOVA, Duncan's multiple range test was used to determine differences among individual means.

Table 1. Levels of CD3, CD4, CD8, CD19, CD4/CD8 and NC cells in the esophageal and gastric cancer patients and control groups

\begin{tabular}{lccc}
\hline & $\begin{array}{c}\text { EC }(\boldsymbol{n}=\mathbf{2 0}) \\
\text { Mean } \pm \text { SE }\end{array}$ & $\begin{array}{c}\text { GC }(\boldsymbol{n}=\mathbf{2 0}) \\
\text { Mean } \pm \text { SE }\end{array}$ & $\begin{array}{c}\text { CG }(\boldsymbol{n}=\mathbf{2 0}) \\
\text { Mean } \pm \text { SE }\end{array}$ \\
\hline CD3 $(\%)$ & $75.135 \pm 1.615 *$ & $68.440 \pm 2.591$ & $68.035 \pm 1.681$ \\
\hline CD4 (\%) & $44.740 \pm 1.853$ & $39.540 \pm 1.729$ & $39.560 \pm 1.614$ \\
\hline CD8 $(\%)$ & $27.550 \pm 2.728 * *$ & $24.775 \pm 2.346$ & $21.055 \pm 1.584$ \\
\hline CD19 (\%) & $10.025 \pm 0.972 *$ & $9.740 \pm 1.463 *$ & $14.950 \pm 1.293$ \\
\hline CD4/CD8 & $2.086 \pm 0.288$ & $2.005 \pm 0.267$ & $2.085 \pm 0.174$ \\
\hline NK cells $(\%)$ & $7.385 \pm 0.852$ & $12.200 \pm 1.447 * * 6.680 \pm 0.9$ \\
\hline
\end{tabular}

EC-esophageal cancer, GC-gastric cancer, $C G$ - control group,

$S E-$ standard error, NK-natural killer, $* p<0.05, * * p<0.01$

\section{Results}

Twenty patients with esophageal cancer (15 females, 5 males), 20 patients with stomach cancer (8 females, 12 males), and 20 healthy individuals ( 12 females, 8 males) as controls were included in the study. The mean age of the patients with esophageal cancer was $59.2 \pm 1.98$ years, patients with stomach cancer $-63.9 \pm 2.45$ years, and control group $55.3 \pm 2.30$ years. There were no statistically significant differences in the age group and gender. Among patients suffering from esophageal cancer, 18 of them $(90 \%)$ had squamous cell carcinoma, and 2 patients $(10 \%)$ had adenocarcinoma. Esophageal cancer was located on the upper third of esophagus in 3 patients (15\%), median third of esophagus in 4 patients $(20 \%)$, and lower third of esophagus in 13 patients (65\%). All stomach cancer patients had adenocarcinoma. As to the localization of cancer in the stomach, 2 of them (10\%) were diffuse, 3 of them (15\%) were in the cardia, 8 of them (40\%) were in the corpus, and $7(35 \%)$ were in the antrum.

The CD3 and CD8 ratios were significantly higher in the patients with esophageal cancer $(p=0.012, p=0.003$, respectively) and the ratio of the NK cells was significantly higher in the patients with stomach cancer $(p=0.001)$ when compared to the control groups. The ratio of CD19 was significantly lower in the two cancer groups $(p=0.031)$. There were no significant differences in the ratio of CD4 and CD4/CD8 between the groups (Table 1). No correlation between the stage of cancer and the ratio of CD cells was detected.

\section{Discussion}

Patients with gastric carcinoma usually have an impaired immune system involving decreased cell-mediated immune response $[11,12]$. Hong et al. found that the ratio of $\mathrm{CD} 3$, CD4, CD8, CD16 and CD19 were lower and the ratio of $\mathrm{CD} 4 / \mathrm{CD} 8$ was higher in the patients with gastric cancer than the control group. These results can be associated with better survival [13]. In another study, the humoral and cellmediated immunity showed variation according to the stage of the cancer [14]. Barbieri et al. found a low CD8 ratio in patients with gastric cancer [15]. On the other hand, Yao et al.'s study showed a high CD8 ratio in patients with gastric cancer. Additionally, they demonstrated that the expression of the human telomerase catalytic subunit (hTERT) was significantly higher in the pre-cancerous lesions of the immunocompromised patients. In the early stage of the gastric cancer, the expression of the hTERT was significantly increased; meanwhile, cellular-mediated immunity was

Table 2. The status of lymphocyte sub-groups compared to the control group

\begin{tabular}{|c|c|c|c|c|c|c|}
\hline & CD3 & CD4 & CD8 & CD4/CD8 & CD19 & Natural killer cells \\
\hline EC & $\uparrow *$ & $\rightarrow$ & $\uparrow * *$ & $\rightarrow$ & $\downarrow *$ & $\rightarrow$ \\
\hline GC & $\rightarrow$ & $\rightarrow$ & $\rightarrow$ & $\rightarrow$ & $\downarrow *$ & $\uparrow * *$ \\
\hline
\end{tabular}

EC-esophageal cancer, GC-gastric cancer, ${ }^{*} p<0.05,{ }^{*} p<0.01$ 
decreased. It was postulated that a telomerase activity would play a critical role in the pathogenesis of gastric cancer [16].

Another study on the correlation between the immunological assessment of the gastric tumor tissue and the tumor prognosis showed that patients with high NK cells had better prognosis. Thus, the postoperative close monitoring is very important for gastric cancer patients with low NK cell infiltration into the tumor tissue [17]. In another similar study, there were no differences in the ratio of T-lymphocytes and NK cells between the patients with gastric cancer and the control group; however, the NKG2D expression of the NK cells was significantly lower in the cancer group. Therefore, it was proposed that the NKG2D in NK might play a critical role in anti-tumor activity [18].

The ratios of $\mathrm{CD}^{+}{ }^{+} \mathrm{CD} 8{ }^{+}$and $\mathrm{CD} 8{ }^{+} \mathrm{CD} 25^{+} \mathrm{T}$ cells were found to be significantly lower in patients with esophageal cancer; however, the ratio of $\mathrm{CD} 4{ }^{+} \mathrm{CD} 25^{+} \mathrm{T}$ cells and the ratio of $\mathrm{CD} 4 / \mathrm{CD} 8$ were found to be significantly higher in the same group [19]. It was also shown that there was a direct relation between the esophageal cancer prognosis and the ratio of CD4 and CD8 cells; though there was no correlation between the ratio of NK cells and the prognosis. In addition, the combined immunotherapy containing active CD4 and CD8 $\mathrm{T}$ cells was shown to be effective in the treatment of the patients with squamous cell esophageal carcinoma [20].

In one study evaluating both patients with gastric cardia and esophageal cancer, the operability of cancer patients was linked to the subgroups of T (CD3) and B (CD19) cells; the high $\mathrm{CD} 4{ }^{+} \mathrm{CD} 25^{+} \mathrm{T}$ cells ratio was correlated with poor prognosis [21].

In our study, the high level of $\mathrm{T}$ lymphocytes in esophageal cancer and the high ratio of the NK cells in the stomach cancer (both T cell and NK cell are parameters for cell-mediated immunity) would suggest that different etiological and immunological mechanisms might play a key role in these cancer types. In addition, the diminished humoral immune responses (insufficient formation of antibodies) in both cancer groups would be evidence for an impaired body response to the tumor cells (Table 2).

In conclusion, understanding of the cancer immunology of esophageal and stomach cancer will provide insight into the pathogenesis of the cancer. Therefore, further and extensive cancer immunology studies should be conducted to develop new diagnostic strategies in patients with upper gastrointestinal tract cancer.

\section{The authors declare no conflict of interests.}

\section{References}

1. Wang RF (2006): Functional control of regulatory T cell and cancer immunotherapy. Semin Cancer Biol 16: 106-114.

2. O'Byrne KJ, Dalgleish AG, Browning MJ, et al. (2000): The relationship between angiogenesis and the immune response in carcinogenesis and the progression of malignant disease. Eur J Cancer 36: 151-169.

3. Franciosi C, Bravo AF, Romano F, et al. (2002): Immunodeficiency in radically operable gastric cancer patients. Hepatogastroenterology 49: 857-859.

4. Martinez ME, Henning SM, Alberts DS (2004): Folate and colorectal neoplasia relation between plasma and dietary markers of folate and adenoma recurrence. Am J Clin Nutr 79: 691-697.

5. Satia JA, Campbell MK, Galanko JA, et al. (2004): Longitudinal changes in lifestyle behaviors and health status in colon cancer survivors. Cancer Epidemiol Biomarkers Prev 13: 1022-1031.

6. Buc E, Kwiatkowski F, Alves A, et al. (2006): Tobacco smoking: a factor of early onset of colorectal cancer. Dis Colon Rectum 49: 1893-1896.

7. Friborg JT, Yuan JM, Wang R, et al. (2007): A prospective study of tobacco and alcohol use as risk factors for pharyngeal carcinomas in Singapore Chinese. Cancer 109: 1183-1191.

8. Foss FM (2002): Immunologic mechanisms of antitumor activity. Semin Oncol 29: 5-11.

9. Wang YX, Su WL, Zhu WX (2004): Correlation of the changes of lymphocyte phenotype to tumor stage and operative pattern of gastric cancer. Ai Zheng 23: 1065-1068.

10. Santos LB, Yamada FT, Scheinberg MA (1985): Monocyte and lymphocyte interaction in patients with advanced cancer. Evidence for deficient IL-1 production. Cancer 56: 1553-1558.

11. Yu QM, Yu CD, Ling ZQ (2012): Elevated circulating CD19+ lymphocytes predict survival advantage in patients with gastric cancer. Asian Pac J Cancer Prev 13: 2219-2224.

12. Shi L, Zhou Q, Wu J, et al. (2012): Efficacy of adjuvant immunotherapy with cytokine-induced killer cells in patients with locally advanced gastric cancer. Cancer Immunol Immunother 61: 2251-2259.

13. Hong WS, Hong SI, Kim CM, et al. (1991): Differential depression of lymphocyte subsets according to stage in stomach cancer. Jpn J Clin Oncol 21: 87-93.

14. Hong WS, Min YI, Son YS, Hong SI (1995): Peripheral blood lymphocyte subsets in patients with stomach cancer. J Korean Med Sci 10: 164-168.

15. Barbieri C, Fujisawa MM, Yasuda CL, et al. (2003): Effect of surgical treatment on the cellular immune response of gastric cancer patients. Braz J Med Biol Res 36: 339-345.

16. Yao XX, Yin L, Sun ZC (2002): The expression of hTERT mRNA and cellular immunity in gastric cancer and precancerosis. World J Gastroenterol 8: 586-590.

17. Ishigami S, Natsugoe S, Tokuda K, et al. (2000): Prognostic value of intratumoral natural killer cells in gastric carcinoma. Cancer 88: 577-583.

18. Wang JY, Dai SQ, Ye WF, et al. (2005): Perioperative changes of cellular immune response of patients with esophageal carcinoma evaluated through detecting AgNOR content in peripheral blood T lymphocytes and T cell subsets. Ai Zheng 24: 861-864.

19. Cho Y, Miyamoto M, Kato K, et al. (2003): CD4+ and CD8+ $T$ cells cooperate to improve prognosis of patients with esophageal squamous cell carcinoma. Cancer Res 63: 1555-1559.

20. Bentdal OH, Frøland SS, Bosnes V, et al. (1996): Alterations in lymphocyte subsets in blood may predict resectability in carcinoma of cardia or oesophagus. Cancer Lett 100: 133-138.

21. Kono K, Kawaida H, Takahashi A, et al. (2006): CD4(+) $\mathrm{CD} 25$ high regulatory $\mathrm{T}$ cells increase with tumor stage in patients with gastric and esophageal cancer. Cancer Immunol Immunother 55: 1064-1071. 